were excellent, with very few eyes giving me problems. I have used the double embedding method as well (to teach students) and have found it to be a poor substitute for the careful, slow processing of eyes using the classic pathway.

I have to admit that many of my colleges did not enjoy handling.the eyes, claiming that they could not get the results which I got. I had many years of practice and an excellent teacher. In addition, my Pathologist had "an understanding" of the lengthy schedule and he, in turn, educated the ophthalmic surgeons and the oncologists to be patient if they wanted good results.

Anne S. van Binsbergen, South African Institute for Medical Research, Chris Hani Baragwanath Hospital in Soweto. South Africa

\section{Ion Milling Thin-Film \& Substrate Cross-Sections}

Commercial ion mills usually have a rotary stage and two ion guns located in opposite directions, like our Gatan 600 in my lab. Ion beams hit the specimen simultaneously from top and bottom at a small angle $\left(0^{\circ}\right.$ to $\left.20^{\circ}\right)$ while the specimen is rotating. But this method sometimes gives poor results when thinning thin-film cross-sectional specimens, because film and substrate material usually have different thinning rates. We found it can be improved by allowing ion beams to hit the specimen only from the back side of the film.

We made a simple modification to our Gatan ion mill holder by making a pair of shields:

1) Cut two, $2 \mathrm{~mm}$ long ceramic rings from an $\mathrm{Al}_{2} \mathrm{O}_{3}$ ceramic tube $10 \mathrm{~mm}$ in diameter and $1 \mathrm{~mm}$ in thickness.

2) Then cut a 90 degree opening on both rings, stick them on the top and bottom plates of the specimen holder, making sure that the openings are pointing at same direction.

When making cross-sectional samples, I used to stick a block of silicon on top of the film. It protects the film and also gives you valuable thickness information when it becomes red (under $20 \mu \mathrm{m}$ thick).

Then the sample is sliced, dimpled or tripod polished. Make sure that the dimpling center or wedge tip is on the silicon side. The final thickness should be less than $20 \mu \mathrm{m}$, but most materials start breaking under $10 \mu \mathrm{m}$.

When mounting the specimen on the holder, put the substrate side towards the opening of the shields and the film side away from the openings. The sputtering angle can be $15^{\circ}$ and the sputtering energy can be $5 \mathrm{KV}$ in the beginning, and $12^{\circ} / 3 \mathrm{KV}$ at the end. While the ion mill stage Is rotating $360^{\circ}$, Ion beams only sputter the specimen from the openings, so milling time is much longer than normal. It used to take me more than 24 hours to finish one specimen. Watch closely when the hole is approaching the film. Check the specimen on a good light microscope to see if there is nice thin area on the film.

This method has worked for dozens of specimens I have made and gave beautiful interface structures on a high resolution microscope. Some ion mill models have rocking or speed control stage, but they are not capable of doing a similar lob.

Chengyu Song, Lawrence Berkeley National Laboratory Berkeley

\section{Internal Size Calibration of Isolated Biological Particles:}

It is often necessary to determine the size of purified viruses, organdies, - macromolecular complexes and other particles. One easy and very accurate way is to use an internal calibration standard that eliminates errors in microscope calibration and effects of hysteresis. We use the method originally published by Dr. Ronald Luftig in 1967 (see below).

Beef liver catalase crystals have a periodic lattice structure which is easy to visualize in the TEM after negative staining. Dr. Luftig calibrated the crystals (available from Sigma and other sources) using copper phthalocyanine crystals and Fullam replica gratings. He determined that the line spacing of the catalase crystals was $91 \pm 3 \AA$ by uranyl acetate (UA) and phosphotungstic acid staining.

The technique for viruses is:

1) A droplet of virus suspension is placed on a formvar coated grid.

2) Grid is inverted onto a droplet of $2 \%$ UA for 90 seconds.

3) Grid is then floated for 2 minutes on a droplet of $0.08 \mathrm{~mL}$ catalase crystal suspension in $5 \mathrm{~mL}$ of $2^{\circ} \%$ UA.

4) Excess stain is drawn off with filter paper and the grid is allowed to air dry.

It is important to check the catalase suspension by negative staining when it is received, since some preparations contain degraded crystals.

This method was preferred to mixing the virus and catalase together prior to staining, in that it gave better stain penetration of the virus.

Measuring particles (viruses macromolecules, etc.) resting on or adjacent to the catalase crystals permits accurate size determination. Catalase is also wonderful for more accurate calibration of TEMs at higher magnifications than can be done with calibration replica gratings.

Luftig, R. and R. Haselkorn. (1967) Morphology of a Virus of Blue-green Algae and Properties of Its Deoxyribonucleic Acid. J. of Virology 1:344 361.

Debby Sherman, Purdue University

\section{SEM Preparation Of Fungi Grown On Agar}

1) Take plug-like samples of the fungi and the agar below it using a widened pipette tip.

2) Place the samples into a small plastic petri dish.

3) Also place in the dish a BEEM capsule lid containing 1 or $2 \%$ osmium tetroxide (in buffer if you wish).

4) Seal and insert this into a larger petri dish.

5) Seal and place the nested dishes in a dark box in a fume hood for 48 hours. Examine every 12 to 15 hours and replace osmium with fresh solution. The fungi should turn black and the agar only a pale gray.

Remove fixative to a waste container with vegetable oil and allow the sample to air $\mathrm{dry}$ for 24 hours. Trim off the agar, glue the disk of fungi to an aluminum stub with double sticky tape, add colloidal silver to the edge of the sample and tape. Sputter-coat with $10 \mathrm{~nm}$ of gold or gold/palladium.

If the fungi has loose threads or fruiting bodies, prepare another SEM stub with double sticky tape and touch it to the surface of another plug to obtained structures fully adhering to the stub - again add a drop of colloidal silver and sputter-coat

This is known as vapor fixation and is very effective for hydrophobic organisms such as fungi.

King, E.J. and M.F. Brown. 1983. A technique for preserving aerial fungal structures for scanning electron microscopy. Can. J. Microbiol. 29:653658.

Quattlebaum, E.C. and G.R. Carner. 1980. A technique for preparing Beauveria spp. for scanning electron microscopy. Can. J. Bot 58:17001703.

Rosemary Walsh.

The Biotechnology Institute for Research and Education, University Park, PA 